Proceedings of the XXXVIII International School and Conference on the Physics of Semiconductors "Jaszowiec" 2009

\title{
AlGaN/GaN Heterostructure FET — Processing and Parameters Evaluation
}

\author{
B. Boratyński*, B. Paszkiewicz, R. Paszkiewicz and M. TŁaczaŁa \\ Faculty of Microsystems Electronics and Photonics, Wrocław University of Technology \\ Janiszewskiego 11/17, 50-370 Wrocław, Poland
}

\begin{abstract}
AlGaN/GaN heterostructure field effect transistors were investigated in terms of microwave and sensor applications. Heterostructure layers grown on sapphire substrates were evaluated using impedance spectroscopy measurements. The 2DEG sheet concentration of $8 \times 10^{12} \mathrm{~cm}^{-2}$ and mobility of $1600 \mathrm{~cm}^{2} /(\mathrm{V} \mathrm{s})$ were obtained. The measured $I-V$ characteristics of the heterostructure field effect transistors devices revealed the saturated drain current $180 \mathrm{~mA} / \mathrm{mm}$ and the gate pinch-off voltage $-2.0 \mathrm{~V}$ with the transconductance $200 \mathrm{mS} / \mathrm{mm}$. The structures have been characterized in microwave frequency range with the measured cut-off frequency of $6 \mathrm{GHz}$ for $1 \mu \mathrm{m}$ gate device. Studies of an AlGaN/GaN heterostructure Schottky diode with a catalytic Pt electrode as a hydrogen gas sensor confirmed high sensitivity of the Schottky barrier on hydrogen adsorption. Differential conductance of the Schottky diode was found to be a convenient parameter to estimate changes of the Schottky barrier height.
\end{abstract}

PACS numbers: 81.05.Hd, 85.30.De, 85.30.Tv

\section{Introduction}

Heterostructure field effect transistors (HFET) based on AlGaN/GaN epitaxial layers find wide range of applications in high frequency, high power electronics due to large breakdown voltage, large saturation electron velocity, unusual robustness and thermal capability of III-N materials. The last two properties directed interest in such heterostructures also into the sensor field. Despite recent progress in HFET design and fabrication methods, which resulted in spectacular parameter values for low-noise HFET [1], the heterostructure growth and device fabrication techniques are far from optimization.

In this paper we report on the developed process technology and measurement techniques applied to $\mathrm{GaN} / \mathrm{AlGaN} / \mathrm{GaN}$ epitaxial structures on sapphire substrates with the aim to fabricate HFETs operating at $1-2 \mathrm{GHz}$ frequency range. Suitability of a given heterostructure sample for any specific application was evaluated by precise $C-V$ measurements within a range of frequencies, which in turn, allowed assessing the main two-dimensional electron gas (2DEG) parameters. The device processing included FET active region definition, ohmic and Schottky contact formation. Some aspects of the electrical contacts parameters of the structure had to be studied and are discussed here, to give insight into the resulting $I-V$ characteristics and microwave parameters of the transistors. The HFET devices were fabricated in two topologies. A two-finger gate topology, which required epitaxial layer active region delineation by plasma

* corresponding author; e-mail: boguslaw.boratynski@pwr.wroc.pl dry etch, and in a round gate topology, that had no need for mesa etch and was more suitable for fast structure fabrication and quick parameters evaluation. The drain and source ohmic contacts were made using $\mathrm{Ti} / \mathrm{Al} / \mathrm{Ni} / \mathrm{Au}$ metallization deposited in a single UHV process. The gate Schottky contact has been obtained using $\mathrm{Ru} / \mathrm{Au}$ or Pt deposited by e-beam technique and the lift-off process. The measured $I-V$ characteristics of the HFET devices revealed the saturated drain current $180 \mathrm{~mA} / \mathrm{mm}$ and the gate-source pinch-off voltage $-2.0 \mathrm{~V}$ with a typical transconductance $200 \mathrm{mS} / \mathrm{mm}$. The HFET structures have been characterized in microwave frequency range revealing the cut-off frequency $f_{T}=2 \mathrm{GHz}$ for $2 \mu \mathrm{m}$ gate length and $f_{T}=6 \mathrm{GHz}$ for $1 \mu \mathrm{m}$ gate length.

Also, results of preliminary studies of AlGaN/GaN heterostructure for hydrogen gas sensor application are included. The Schottky junction barrier height dependence on hydrogen concentration in a sampled gas volume was investigated by measurement of the differential conductance of the Schottky diode. High sensitivity of the diode with a catalytic Pt gate electrode was confirmed experimentally.

\section{AlGaN/GaN epitaxial structure evaluation}

Two different types of 2DEG heterostructures were investigated. First, a heterostructure with a single quantum well (QW) and the following layer sequence: undoped cap-GaN(5 nm)/n-doped $\mathrm{Al}_{x} \mathrm{Ga}_{1-x} \mathrm{~N}$ $(25 \mathrm{~nm}, x=0.3) /$ undoped-GaN buffer $(1550 \mathrm{~nm}) /$ low temperature-GaN $(20 \mathrm{~nm}) /$ sapphire substrate and second, a double heterostructure with an optimised quantum well, as above, and underlying buried AlGaN 


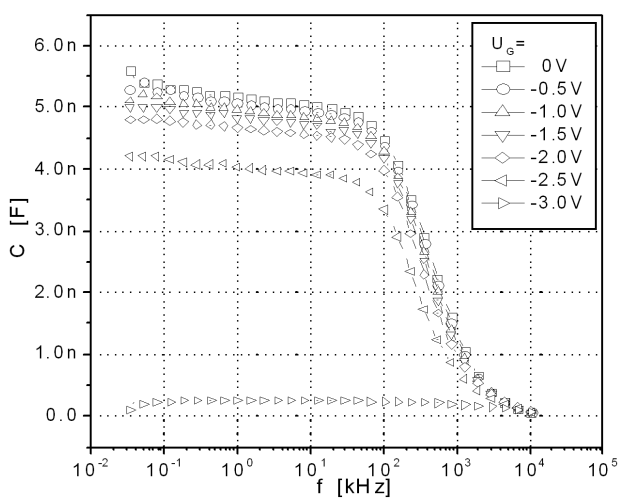

Fig. 1. Capacitance of the Hg-probe Schottky junction vs. frequency for the voltage bias from $0 \mathrm{~V}$ to $-3 \mathrm{~V}$.

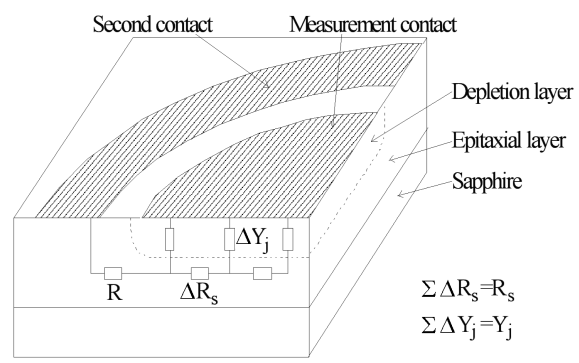

Fig. 2. Schematics of $C-V$ measurements using mercury probe and elements of the equivalent distributed model.

layer with the following layer sequence: undoped cap-GaN $(5 \mathrm{~nm}) / n$-doped $\mathrm{Al}_{x} \mathrm{Ga}_{1-x} \mathrm{~N}(25 \mathrm{~nm}, \quad x=$ $0.3) /$ undoped-GaN(350 nm)/ $\mathrm{Al}_{x} \mathrm{Ga}_{1-x} \mathrm{~N}(10 \mathrm{~nm}, x=$ $0.3)$ / undoped-GaN buffer(1200 nm)/low temperature-GaN(20 nm)/sapphire substrate. The purpose of introducing the second AlGaN layer was improving the semi-insulating properties of the undoped GaN layer, and thus minimizing influence of the substrate on the 2DEG parameters in the upper interface.

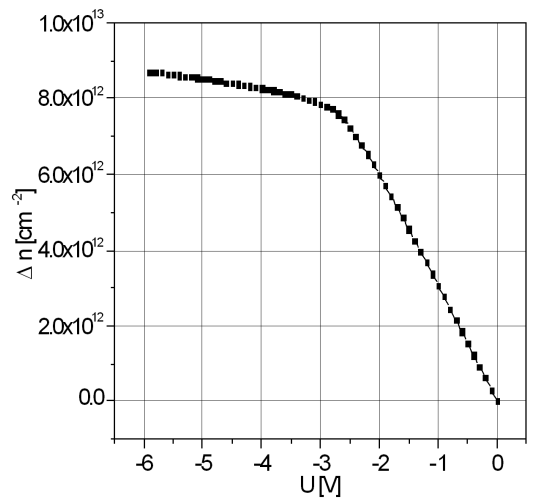

Fig. 3. Incremental 2DEG sheet charge versus the voltage bias in the single QW sample.

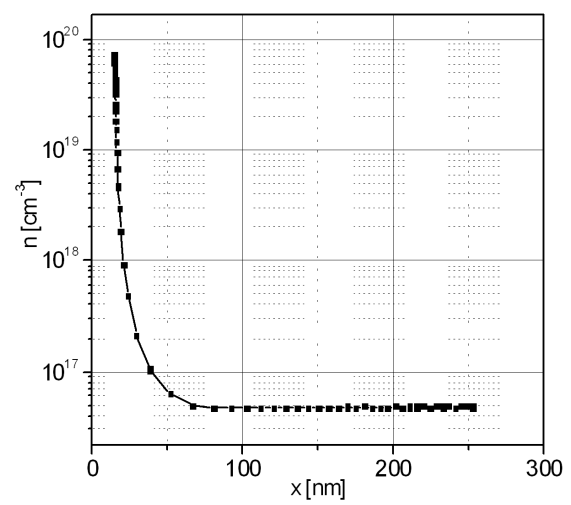

Fig. 4. Free electron distribution in the single QW sample.

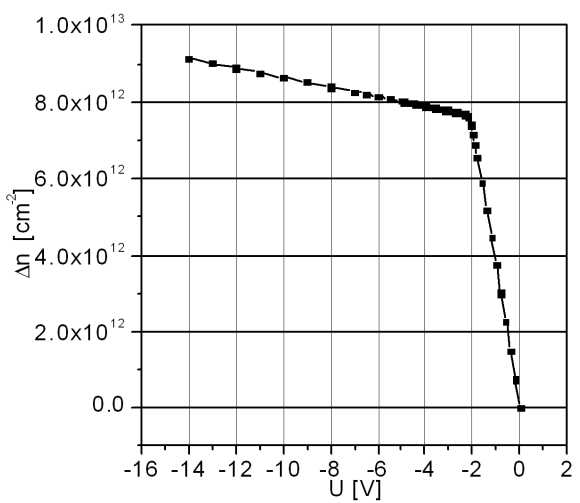

Fig. 5. Incremental 2DEG sheet charge versus the voltage bias in the double $\mathrm{QW}$ sample.

Electrical properties of the $\mathrm{Al}_{x} \mathrm{Ga}_{1-x} \mathrm{~N} / \mathrm{GaN}$ heterostructures were determined by the frequency dependent $C-V$ measurements (impedance spectroscopy) performed using an impedance analyser and a two-contact mercury probe. The capacitance was measured in the range of frequencies from $20 \mathrm{~Hz}$ to $10 \mathrm{MHz}$ and the re-

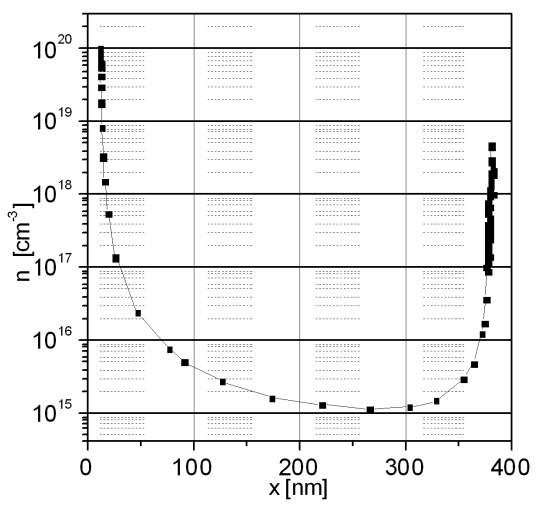

Fig. 6. Free electron distribution in the double QW sample. 
sults were fitted to the distributed element equivalent circuit model. Results of the capacitance versus frequency measurements for different reverse bias of the $\mathrm{Hg}$-probe Schottky contact to the investigated sample are shown in Fig. 1. A dedicated distributed element equivalent circuit model regarding the series resistances and the Schottky junction capacitance and conductance was applied [2]. In Fig. 2 schematics of the measurement principle and major components of the equivalent model are shown. The model allowed proper interpretation of the junction capacitance versus bias voltage dependence. As a result, electron concentration and the electron mobility of the 2DEG in the AlGaN/GaN quantum well were precisely defined and this provided insight into the expected parameters of the devices to be fabricated. As seen from the $C-f$ ( $U$-parameter) plots in Fig. 1, the capacitance reveals slight dependence on the measurement frequency, which could be related to relaxation phenomena in the 2DEG layer. The relaxation source could be located at the surface states or in the bulk deep trap levels. In HFETs the relaxations are reflected as hysteresis-like $I-V$ characteristics. The effect is known as the drain current lag and it is particularly cumbersome in large signal operation regime.

In Fig. 1 one can notice that carrier depletion in the 2DEG channel starts for the gate voltage of $-2.0 \mathrm{~V}$ and at $-3.0 \mathrm{~V}$ the channel is completely depleted. Calculated 2DEG sheet charge variation during the channel depletion due to the applied bias is shown in Fig. 3. In the presented single QW heterostructure, the depleted sheet charge accounted to almost $8 \times 10^{12} \mathrm{~cm}^{-2}$ at the pinch off $-2.8 \mathrm{~V}$. This represented total 2DEG concentration $8 \times 10^{19} \mathrm{~cm}^{-3}$ at the AlGaN/GaN interface, i.e. at the sample depth of $30 \mathrm{~nm}$. The overall electron distribution in the sample is shown in Fig. 4.

In the double QW heterostructure (with the buried AlGaN layer) the depleted charge in the top QW was calculated to be $7.5 \times 10^{12} \mathrm{~cm}^{-2}$ at the pinch-off voltage $-2.3 \mathrm{~V}$, as shown in Fig. 5 and the 2DEG concentration was evaluated to be $10^{20} \mathrm{~cm}^{-3}$ (Fig. 6). In the bottom side QW the calculated carrier concentration distribution, also shown in Fig. 6, has reached level of $5 \times 10^{18} \mathrm{~cm}^{-3}$ at the maximum applied bias of $-14 \mathrm{~V}$, which corresponded to the sample depth of $350 \mathrm{~nm}$. Calculated from the measurements drift electron mobility in 2DEG was $1600 \mathrm{~cm}^{2} /(\mathrm{Vs})$.

\section{HFET device fabrication and characterization}

Two different types of heterostructure FET were investigated. For high frequency tests and applications a standard two-finger gate $(2 \times 125 \mu \mathrm{m})$ layout was used and mesa dry etch definition of the FET active region was applied. A top view of the fabricated device is shown in Fig. 7. For testing high power and sensor applications a circular contact layout topology has been used to avoid the need for mesa etching process. In this case, the source electrode eliminating possible parasitic shunt drain current, which would flow otherwise, surrounded the inner drain contact and the gate electrode. The circular HFET topology and test structures are shown in Fig. 8. The total channel width was $400 \mu \mathrm{m}$ or $700 \mu \mathrm{m}$ for the round structures. In both topologies the nominal gate length was $2 \mu \mathrm{m}$ and the drain to gate and source to gate separation was $1.5 \mu \mathrm{m}$.

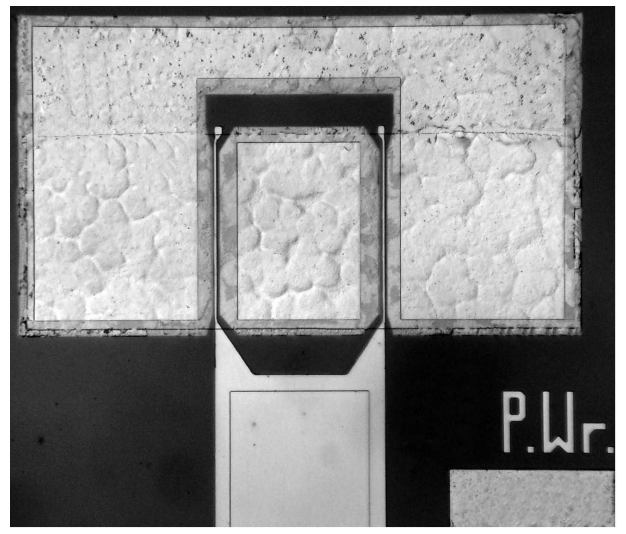

Fig. 7. Standard topology - two-finger gate HFET device.

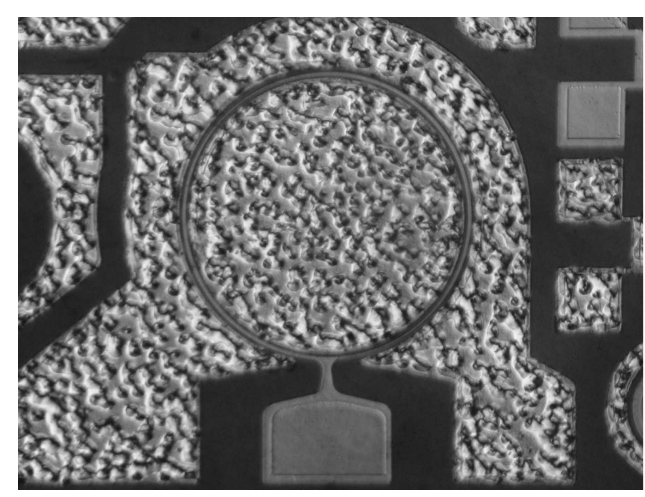

Fig. 8. Circular topology HFET device.

In case of mesa etching for two-finger gate devices, reactive-ion etching (RIE) technique was used to obtain $100 \mathrm{~nm}$ deep mesa etch. The drain and source ohmic contacts were made using $\mathrm{Ti} / \mathrm{Al} / \mathrm{Ni} / \mathrm{Au}(10 / 100 / 40 / 100 \mathrm{~nm})$ metallization deposited in a single UHV process. After the lift off, the wafers were rapid thermal annealing (RTA) processed for contact annealing in nitrogen ambient at $850^{\circ} \mathrm{C}$ for $45 \mathrm{~s}$. To obtain good contact surface morphology and low resistivity, a second metallization of $\mathrm{Ti} / \mathrm{Au}(50 / 200 \mathrm{~nm})$ was applied. The resulting contact resistance was of the order of $10^{-5} \Omega \mathrm{cm}^{2}$. The contact metallization sheet resistance was $1.0 \Omega / \mathrm{sq}$. This was not satisfactory for assuring small series resistance of the drain and source and further studies including $n^{+}$-type subcontact silicon ion implantation and contact electroplating were already initiated.

The gate Schottky contact has been made using standard $\mathrm{Ni} / \mathrm{Pt} / \mathrm{Au}$ metallization and a newly developed met- 
allization based on $\mathrm{Ru} / \mathrm{Au}$ bilayer. Both types of contact metallization were deposited by e-beam evaporation and the lift-off process. Ruthenium based contacts were tested by thermal processing at temperatures $200^{\circ} \mathrm{C}$ to $500{ }^{\circ} \mathrm{C}$ for barrier height and leakage current improvement. It showed up that annealing at temperature $500^{\circ} \mathrm{C}$ for 2 min resulted in the highest value of the Schottky barrier of $1.1 \mathrm{eV}$ obtained on the $n$-doped $\left(10^{17} \mathrm{~cm}^{-3}\right)$ GaN sample. The leakage current $10^{-6} \mathrm{~A} \mathrm{~cm}^{-2}$ was measured at $-15 \mathrm{~V}$ reverse bias. The completed devices were surface protected by a polyamide layer. The output, $I_{\mathrm{D}}-\mathrm{U}_{\mathrm{DS}}$ characteristics for the standard topology HFET are presented in Fig. 9 and for the round topology HFET in Fig. 10.

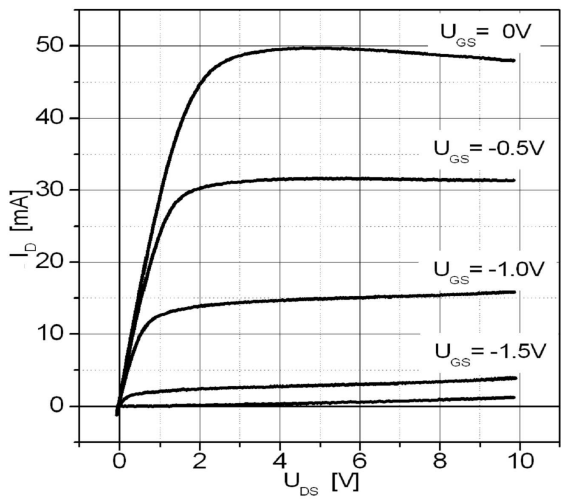

Fig. 9. Drain $I-V$ characteristics of the two-finger gate HFET device for gate voltage from $0 \mathrm{~V}$ to $-2 \mathrm{~V}$.

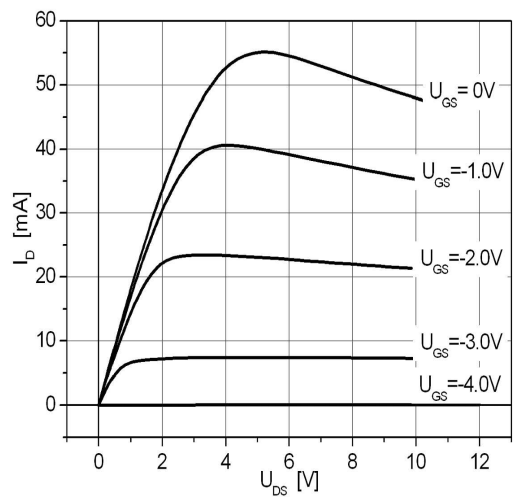

Fig. 10. Drain $I-V$ characteristics of the circular gate HFET for gate voltage from $0 \mathrm{~V}$ to $-4 \mathrm{~V}$.

Microwave parameters of the selected HFETs have been measured on-wafer using Agilent N5230A vector analyser and coplanar Picoprobe point contact probes. $S$-parameters, transducer gain $\left(G_{\mathrm{T}}\right)$, maximum available gain (MAG), gain stability factor and gain linearity have been evaluated. The results obtained for a standard topology HFET with two-finger gate width of $2 \times 125 \mu \mathrm{m}$ are shown in Fig. 11 to Fig. 14. In Fig. 11 power gain, $G_{\mathrm{T}}$ calculated from $\boldsymbol{S}$-parameters is presented. The plot re- veals that the cut-off frequency $f_{T}$ was just above $2 \mathrm{GHz}$. Maximum available gain dependence on signal frequency presented in Fig. 12 shows that below $200 \mathrm{MHz}$ the HFET is potentially unstable, which is typical for microwave HFETs. This is confirmed by measurements presented in Fig. 13, where the stability factor $k$ is lower than unity for frequencies below $200 \mathrm{MHz}$.

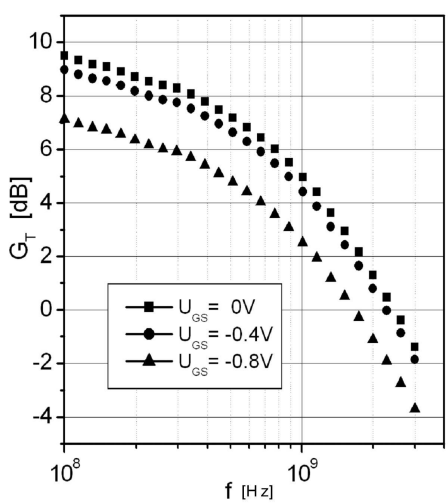

Fig. 11. Gain, $G_{\mathrm{T}}$ versus frequency for different gate to source voltage bias.

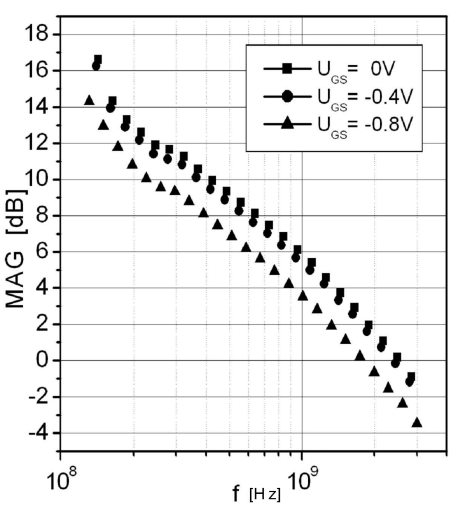

Fig. 12. MAG versus frequency for different gate to source voltage bias points.

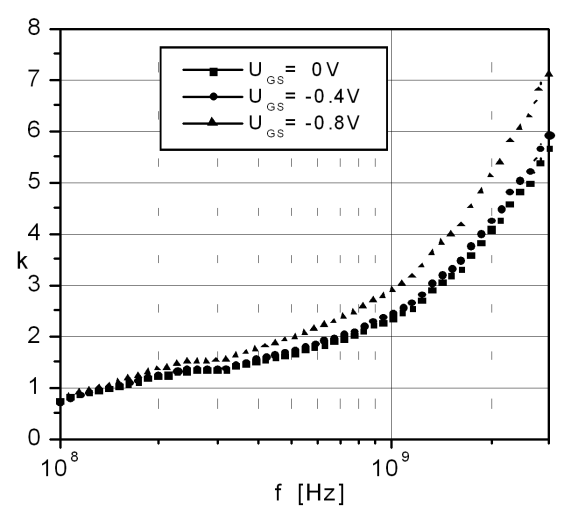

Fig. 13. Stability factor versus the frequency. 


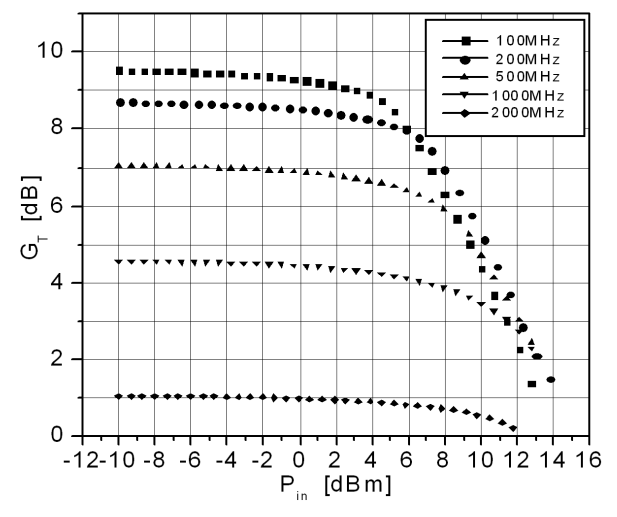

Fig. 14. Power gain versus input power for different signal frequency.

Power gain linearity has been measured within range of input signal power $-10 \mathrm{dBm} \div+13 \mathrm{dBm}$. From the gain versus input power level dependence depicted in Fig. 14 the $1 \mathrm{~dB}$ compression point can be found. The output power at $1 \mathrm{~dB}$ compression point $\left(P_{-1 \mathrm{~dB}}\right)$ exceeded $13 \mathrm{dBm}(20 \mathrm{~mW})$ at the signal frequency of $1 \mathrm{GHz}$ and it was $12.3 \mathrm{dBm}(17 \mathrm{~mW})$ for the signal frequency of $2 \mathrm{GHz}$. These results were expected, since the relevant parameter, the gate length was $2 \mu \mathrm{m}$. In the case of a HFET device with gate length of $1 \mu \mathrm{m}$, fabricated on an identical heterostructure sample, essential improvement in $f_{T}$ value of $6 \mathrm{GHz}$ has been obtained.

\section{Sensor application}

The fabricated AlGaN/GaN/GaN heterostructures were tested also for gas sensor application. Since the 2 DEG channel is located very near $(30 \mathrm{~nm})$ from the surface, any change in the surface charge influences the carrier concentration of the $2 \mathrm{DEG}$. In result, variations of the drain current in the saturated region of the drain $I-V$ characteristics or change of the FET on-resistance in the linear region of the $I-V$ characteristics can be measured. To utilize these effects a virtual or catalytic gate (chemical gate) could be implemented in the HFET topology, where the adsorption of the sensed gas or liquid molecules could take place. The adsorbed molecules change the surface charge and thus, the Schottky barrier height (SBH) at the virtual gate. Especially suitable for this application is a FATFET design (FET with large gate area) presented in Fig. 15.

To study the gas adsorption effects, Schottky diodes with a catalytic $10 \mathrm{~nm}$ thick Pt contacts were fabricated on the $30 \mathrm{~nm}$ undoped $\mathrm{Al}_{0.16} \mathrm{Ga}_{0.84} \mathrm{~N}$ layer grown on $2 \mu \mathrm{m}$ thick undoped GaN layer deposited on a low temperature GaN buffer. The heterostructure was Ga-polar and the 2DEG was present at the AlGaN/GaN interface. The sheet concentration of the 2DEG was determined to be $3.7 \times 10^{12} \mathrm{~cm}^{-2}$. The ohmic contacts were made using $\mathrm{Ti} / \mathrm{Al} / \mathrm{Ni} / \mathrm{Au}$ metallization deposited in a single UHV process followed by rapid thermal annealing (RTP). Fi-

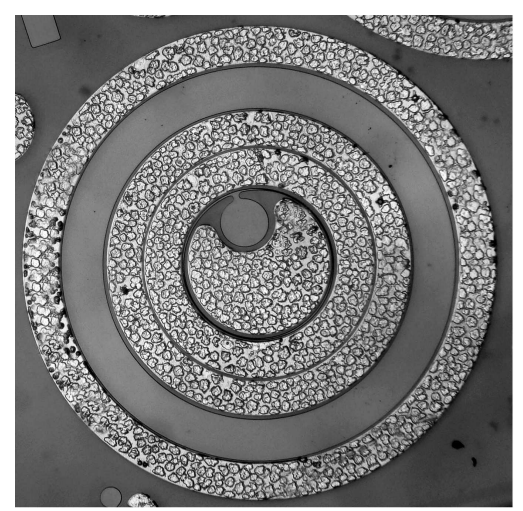

Fig. 15. FATFET heterostructure sensor.

nally, the $\mathrm{Ti} / \mathrm{Au}(50 \mathrm{~nm} / 200 \mathrm{~nm})$ was deposited on top of the ohmic contacts and on the outer ring of the Schottky $\mathrm{Pt}$ electrode to decrease the parasitic series resistance and to allow the point probe measurements. A top view of the sensor diode is shown in Fig. 16.

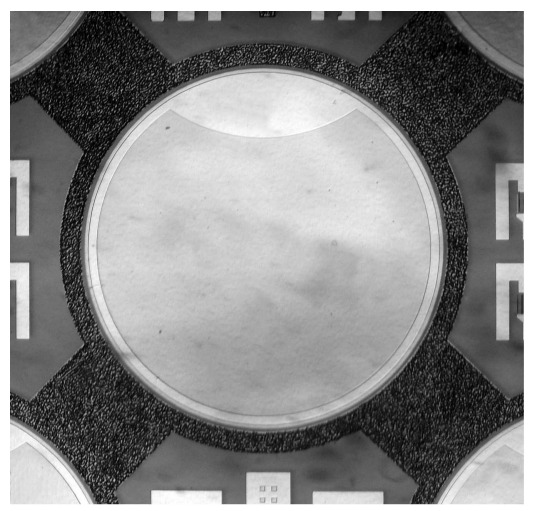

Fig. 16. Catalytic Pt gate Schottky diode sensor.

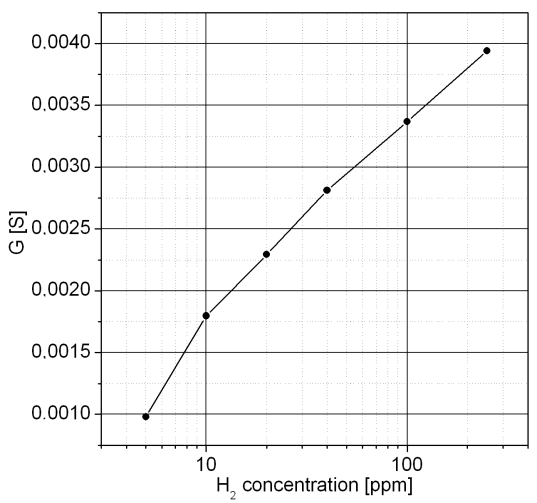

Fig. 17. The Schottky junction conductance at zero bias for different concentrations of $\mathrm{H}_{2}$.

The diodes were tested as hydrogen gas sensor at the temperature range from $-20^{\circ} \mathrm{C}$ to $120^{\circ} \mathrm{C}$ and the hydro- 


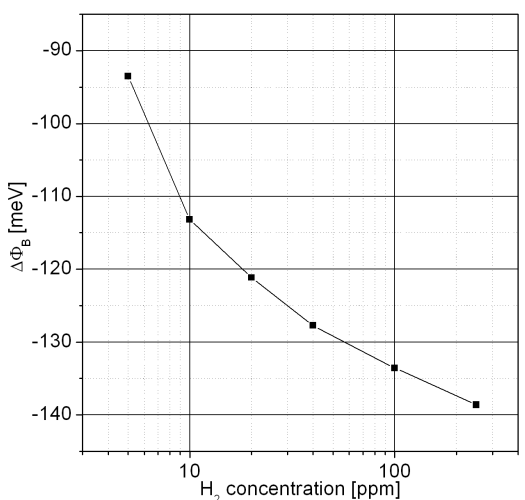

Fig. 18. Relative change of the Schottky barrier height for different concentrations of $\mathrm{H}_{2}$.

gen volume concentration from $0 \mathrm{ppm}$ to $250 \mathrm{ppm}$ in nitrogen [3]. The differential conductance of the Schottky contact $G_{\mathrm{d}}=I_{0} /(k T / q)$, taken at zero bias was selected as a measured parameter. From the conductance the Schottky thermionic current could be calculated, which in turn is strongly dependent on the SBH. Biasing at $0 \mathrm{~V}$ allowed avoiding the influence of the leakage current, which usually occurs in large area and large perimeter junction. The measurements were performed at $1 \mathrm{kHz}$ using impedance analyzer. The dynamics of the conductance changes was evaluated for the various hydrogen concentrations in the gas chamber. It was observed that after each measurement cycle the nitrogen flush caused return of the conductance to the initial value. Also, for each cycle, the dynamics of conductance decrease was the same and comparable with the dynamics of conductance increase. The results of conductance changes as a function of hydrogen concentration at the chamber are presented in Fig. 17. This dependence is evidently logarithmic except the point obtained for $5 \mathrm{ppm}$ of hydrogen. This deviation may be caused by not reaching the equilibrium condition for such low hydrogen concentration. The evaluation of the decrease of the $\mathrm{SBH}$ value, as a function of hydrogen concentration is presented in Fig. 18. It was observed that the hydrogen concentration change from 0 to $5 \mathrm{ppm}$ caused significant decrease of the $\mathrm{SBH}$ by $95 \mathrm{meV}$.

Hydrogen adsorption dependent variations of the SBH value were determined also from the Arrhenius plots of conductance obtained from the temperature measurements. A good agreement with the previous results was obtained with the value of $89 \mathrm{meV}$ decrease of the $\mathrm{SBH}$ resulting from the $5 \mathrm{ppm}$ hydrogen concentration in nitrogen.

\section{Conclusions}

$\mathrm{GaN} / \mathrm{AlGaN} / \mathrm{GaN}$ epitaxial structures on sapphire substrates were evaluated by impedance spectroscopy measurements. It allowed assessing the 2DEG parameters: carrier sheet concentration of $8 \times 10^{12} \mathrm{~cm}^{-2}$, carrier mobility $1600 \mathrm{~cm}^{2} /(\mathrm{Vs})$ and the pinch-off voltage $-2 \mathrm{~V}$. Using the substrates, HFETs with the gate length $2 \mu \mathrm{m}$ were fabricated and microwave parameters with the cut-off frequency $f_{T}$ of $3 \mathrm{GHz}$ were measured. Improvement in ohmic contact processing and reduction of the gate length to $1 \mu \mathrm{m}$ resulted in much better values of the relevant HFET parameters with the measured $f_{T}$ of $6 \mathrm{GHz}$. Microwave parameters of the HFETs were defined including power gain of $8 \mathrm{~dB}$ at $1 \mathrm{GHz}$ for $1 \mu \mathrm{m}$ HFET device. In a separate study, application of the AlGaN/GaN heterostructure to hydrogen gas sensors was investigated. Differential conductance of the Schottky diode with a catalytic Pt anode was found to be a convenient parameter to estimate changes in the Schottky barrier height caused by variation of hydrogen concentration in the sampled gas.

\section{Acknowledgments}

This work has been supported in a part by the Ministry of Science and Higher Education under the grants no. R0201802, PBZ-MEiN-6/2/2006, NN 515360436, NN 315360436 , by the European Union within European Regional Development Fund Grant Innovative Economy (POIG.01.01.02-00-008/08) and by Wrocław University of Technology statutory grant.

\section{References}

[1] H. Sun, A.R. Alt, H. Benedickter, C.R. Bolognesi, IEEE Electron Device Lett. 30, 107 (2009).

[2] B. Paszkiewicz, J. Cryst. Growth 230, 590 (2001).

[3] B. Paszkiewicz, R. Paszkiewicz, W. Macherzynski, J. Prazmowska, A. Szyszka, M. Wosko, M. Krasowska, A. Stafiniak, M. Tlaczala, in: Proc. 13th EWMOVPE 2009, Ulm 2009, C-12, p. 253. 